\title{
Axisymmetric polydimethysiloxane microchannels for in vitro hemodynamic studies
}

\author{
Rui Lima ${ }^{1,2,7}$, Mónica S N Oliveira ${ }^{3}$, Takuji Ishikawa ${ }^{4}$, Hirokazu Kaji ${ }^{4}$, \\ Shuji Tanaka ${ }^{5}$, Matsuhiko Nishizawa ${ }^{4}$ and Takami Yamaguchi ${ }^{6}$ \\ ${ }^{1}$ Department of Mechanical Technology, ESTiG, Bragança Polyt., C. Sta. Apolonia, 5301-857 \\ Bragança, Portugal \\ ${ }^{2}$ CEFT, Faculdade de Engenharia da Universidade do Porto, Rua Dr. Roberto Frias, 4200-465 Porto, \\ Portugal \\ ${ }^{3}$ Departamento de Engenharia Química, CEFT, Faculdade de Engenharia da Universidade do Porto, \\ Rua Dr Roberto Frias, 4200-465 Porto, Portugal \\ ${ }^{4}$ Department of Bioengineering and Robotics, Graduate School of Engineering, Tohoku University, \\ 6-6-01 Aoba, 980-8579 Sendai, Japan \\ ${ }^{5}$ Department of Nanomechanics, Graduate School of Engineering, Tohoku University, 6-6-01 Aoba, \\ 980-8579 Sendai, Japan \\ ${ }^{6}$ Department of Biomedical Engineering, Graduate School of Biomedical Engineering, \\ Tohoku University, 6-6-01 Aoba, 980-8579 Sendai, Japan \\ E-mail: ruimec@ipb.pt
}

Received 23 May 2009

Accepted for publication 24 August 2009

Published DD MMM 2009

Online at stacks.iop.org/BF/1/000000

\begin{abstract}
The current microdevices used for biomedical research are often manufactured using microelectromechanical systems (MEMS) technology. Although it is possible to fabricate precise and reproducible rectangular microchannels using soft lithography techniques, this kind of geometry may not reflect the actual physiology of the microcirculation. Here, we present a simple method to fabricate circular polydimethysiloxane (PDMS) microchannels aiming to mimic in vivo microvascular environment and suitable for state-of-the-art microscale flow visualization techniques, such as confocal $\mu \mathrm{PIV} / \mathrm{PTV}$. By using a confocal $\mu \mathrm{PTV}$ system individual red blood cells (RBCs) were successfully tracked trough a $75 \mu \mathrm{m}$ circular PDMS microchannel. The results show that RBC lateral dispersion increases with the volume fraction of RBCs in the solution, i.e. with the hematocrit.
\end{abstract}

(Some figures in this article are in colour only in the electronic version)

\section{Introduction}

Along the years, various experimental studies have been performed on glass microchannels (Chien et al 1984, Goldsmith and Turitto 1986) in an effort to understand the blood flow behavior in microcirculation. These studies used a variety of experimental techniques, such as flow visualization, micro-particle image velocimetry ( $\mu \mathrm{PIV})$ and micro-particle tracking velocimetry ( $\mu \mathrm{PTV})$, and have enabled

\footnotetext{
7 Author to whom any correspondence should be addressed.
}

the observation of several flow phenomena that are also present in real blood vessels. Among the most remarkable phenomena observed both in vivo and in glass capillaries are the well-known Faharaeus effect and Faharaeus-Lindqvist effect, which are recognized to depend strongly on the microtube diameter (Chien et al 1984). Although the flow through glass capillaries presents certain similarities to in vivo microcirculation, it is also clear that this kind of rigid microchannels differ from real microvessels in several aspects related to the elasticity of the walls, the role of the irregularly shaped endothelial surface and the effect of 
branches and asymmetrical structure of microvessels. Thus, it is not surprising that several studies of blood flow in glass microtubes and in microvessels have yielded conflicting results with respect to flow resistance (Pries et al 1994) and deformability of red blood cells (RBCs) (Suzuki et al 1996). In the former case, the authors reported that the apparent viscosity was higher in vivo than in glass tubes, whereas in the latter it was found that RBCs deform less in real microvessels than in glass capillaries. Despite the efforts to explain the discrepancies observed between in vivo and in vitro results, these have not yet been fully understood.

It is obvious that the conflicting quantitative results arising from in vivo/in vitro experiments cannot be explained considering the flow in straight, rigid glass microchannels alone (Lima et al 2007, 2009). Various researchers have tried to overcome the limitations of glass capillaries by using conventional soft lithography techniques (McDonald et al 2000), which have the ability to generate precise, reproducible and versatile rectangular polydimethysiloxane (PDMS) microchannels (Oliveira et al 2006, 2008, Lima et al 2008a). Such channels have been used to study blood flow in complex geometries, including branched structures, in an effort to mimic human arterioles and capillary networks (Shevkoplyas et al 2003, Fujiwara et al 2009). However, it must be emphasized that these channels have a rectangular cross-section, in contrast to real vessels which have a circular cross-section when subject to no external pressure, and thus the blood flow behavior through them may be significantly different from that of living microvessels.

The flow behavior of blood cells in microvessels is not only dependent on the vessel geometry but several other biochemical and biophysical factors may also contribute to the rheological characteristics of blood. Due to the inability to culture living cells in glass microchannels, several researchers have been using parallel plate flow chambers (Brown and Larson 2001) to investigate the cellular and molecular events occurring between the blood cells and the endothelium. Unfortunately, the flow conditions provided by these microchannels differ markedly from those in microvessels. Recently, Kaji et al $(2006,2007)$ used an innovative cellular micropatterning technique based on the electrochemical method to successfully culture endothelial cells on the surfaces of rectangular PDMS microchannels and silicone tubing. This is a significant advance, but is once more limited by the different cross-sections of the PDMS channels and the real blood vessels.

It is therefore clear that our ability to represent in vivo blood vessels in a more realistic way hinges on the development of circular PDMS microchannels. Such a set-up would go beyond rigid glass capillaries, would not suffer from the problems associated with rectangular PDMS channels, would allow for the use of microscale flow visualization methods, such as conventional and confocal $\mu \mathrm{PIV} / \mu \mathrm{PTV}$, and would potentially permit the culture of live endothelial cells on the channel surface. Wang et al (2007) have proposed a technique to build microvessel scaffolds with circular crosssection channels by bonding two separate PDMS pieces containing channels with a semi-circular cross-section. These 'half-channels' were produced by soft-lithography using a mold containing a convex structure, which was fabricated by a photolithographic technique combined with photoresist melting. This technique allows for the fabrication of complex microstructures but requires expensive equipments and a demanding alignment step and a plasma bonding step to ensure a correct adhesion between the two parts of the channel.

Here, we report a simple and inexpensive method to manufacture circular PDMS microchannels, conceptually similar to a technique reported by Takeuchi et al (2005) to build axisymmetric microfluidic geometries for droplet production, although here we achieve circular channels of diameter one order of magnitude lower. Unlike Wang et al (2007), our microchannels are fabricated from a single piece of PDMS, thus avoiding alignment and bonding problems altogether and ensuring a good overall quality. We demonstrate that our microchannels are suitable for performing flow visualization of labeled and non-labeled RBCs. In addition, we show the ability of our confocal $\mu$ PTV system to follow individual RBCs flowing through a $75 \mu \mathrm{m}$ PDMS microchannel manufactured by the proposed technique. In particular, we quantify the RBC lateral dispersion coefficient $\left(D_{\mathrm{yy}}\right)$ at different hematocrit $(\mathrm{Hct})$.

\section{Materials and methods}

\subsection{Fabrication of the circular PDMS microchannel}

The circular PDMS microchannels were fabricated by a wire casting technique, which can be performed at low cost in a straightforward manner. Specifically, the fabrication process, which is outlined in figure 1, was as follows.

(a) The master was first fabricated using an acrylic base, a needle, screws, nuts and several washers or o-rings. The precision wires were inserted into the needle and then stretched by rotating the screw. Depending on the diameters and characteristics required, the materials tested were silicon, stainless steel, polyester or aluminum (Riken).

(b) The PDMS prepolymer was prepared by mixing a commercial prepolymer and catalyst (Silpot 184; Dow Corning, USA) at a weight ratio of 10:1. The PDMS mixture was poured into the mold master and baked on a hot plate for about $2 \mathrm{~h}$ at low temperature $\left(60^{\circ} \mathrm{C}\right)$. Both master and PDMS were then cooled to room temperature and the PDMS was peeled from the master.

(c) The PDMS containing the needle and the precision wire was then immersed in ethanol for periods of $1-4 \mathrm{~h}$ at $4{ }^{\circ} \mathrm{C}$. This procedure is extremely important in order to allow a smooth extraction of the precision wire.

(d) Finally, the needle and wire were pulled out gently and the inlet and outlet ports were introduced in the PDMS device.

\subsection{Working fluids}

The working fluids used in this study were Dextran 40 (Dx40; Otsuka Medicine, Tokyo, Japan) containing 3\% (3Hct), 

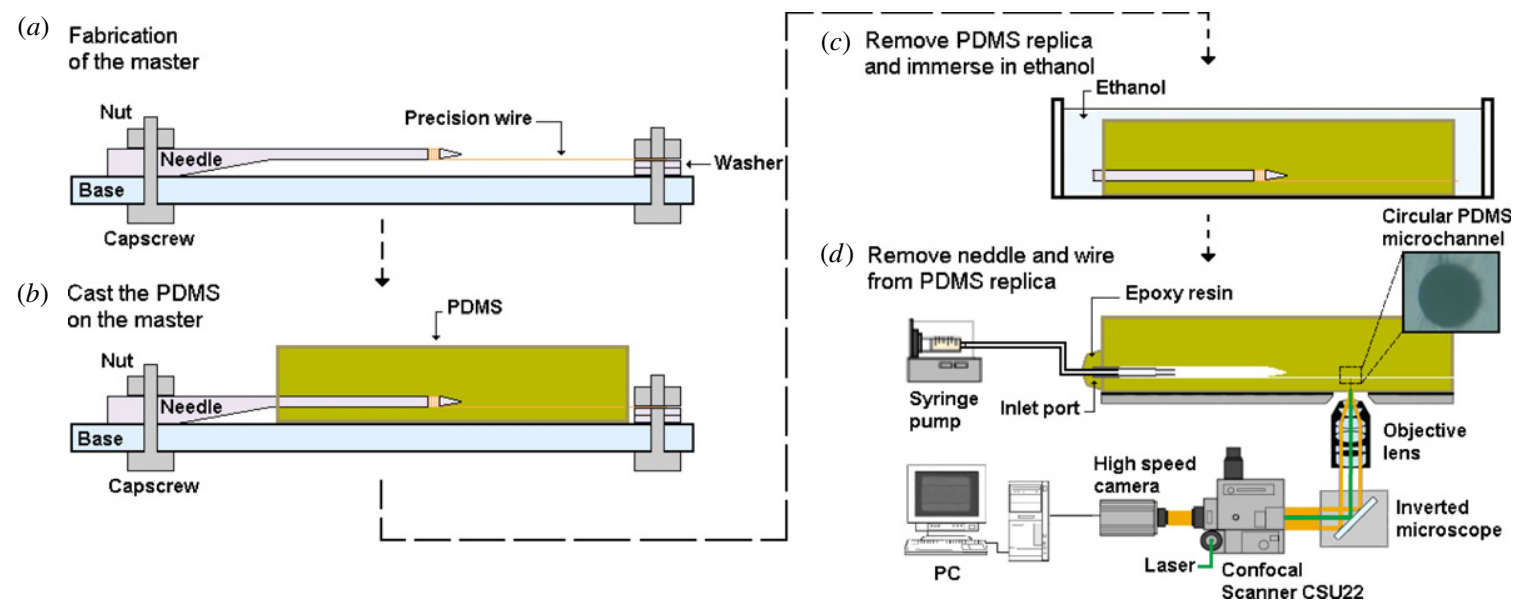

Figure 1. Schematic diagram describing the fabrication procedure of circular PDMS microchannels and micro-visualization technique used to measure the blood flow through the microchannel.

$13 \%$ (13Hct) and $23 \%$ (23Hct) of human RBCs. The RBCs used were fluorescently labeled with a lipophilic carbocyanine derivative dye, chloromethylbenzamido (CM-Dil, C-7000; Ex/Em: 553/570 nm; Molecular Probes, Eugene, OR, USA). Detailed procedures for collecting and labeling RBCs are given elsewhere (Lima et al 2009). All procedures were carried out in compliance with the Ethics Committee on Clinical Investigation of Tohoku University.

\subsection{Confocal $\mu P T V$}

The confocal $\mu$ PTV system used in the present study consists of an inverted microscope (IX71, Olympus, Japan) combined with a confocal scanning unit (CSU22, Yokogawa, Japan), a diode-pumped solid state (DPSS) laser (Laser Quantum Ltd, England) with an excitation wavelength of $532 \mathrm{~nm}$ and a highspeed camera (Phantom v7.1, USA).

The circular PDMS microchannel was placed on top of the stage of the inverted microscope, which was kept at $37^{\circ} \mathrm{C}$ by means of a thermo-plate controller (Tokai Hit, Japan). The flow rate of the fluids was controlled using a syringe pump (KD Scientific Inc., USA).

For the particle tracking measurements, fluorescently labeled RBCs were used and the confocal images were recorded at the center plane of the microchannels, using a piezodriver system and RT3D software (Yokogawa Corporation). A series of $x-y$ confocal images were captured using a $20 \times$ objective lens (1.6 zoom) with a numerical aperture of 0.75 at a rate of 100 frames $\mathrm{s}^{-1}$ with an exposure time of $9.4 \mathrm{~ms}$. The recorded images, which have a resolution of $640 \times 480$ pixels, were then transferred to a computer and evaluated in Image J (NIH) (Abramoff et al 2004) using the manual tracking MtrackJ plugin (Meijering et al 2006). Using this software, we are able to track single RBCs flowing through the center plane of the microchannel and thus determine their position in the microchannel as a function of time. The fluorescently labeled RBCs were tracked for periods of time up to $0.5 \mathrm{~s}$, with a temporal resolution of $10 \mathrm{~ms}$. The bright centroid of each selected RBC was automatically computed in successive images and the $x$ and $y$ positions were obtained.
Table 1. Shape factor values of the microfabricated axisymmetric channels.

\begin{tabular}{ll}
\hline Shape factor & Range \\
\hline Roundness & $0.95-0.99$ \\
Aspect ratio & $0.95-1.00$ \\
Convexity & $0.97-1.00$ \\
\hline
\end{tabular}

It should be noted that by using this technique we were able to track the labeled RBCs even when two moving cells were in close proximity. After obtaining the sequence of $x$ and $y$ positions, and knowing the time between frames, we were able to determine physical quantities such as velocity, radial displacement and dispersion coefficient. Further information on the experimental set-up used in the present study can be found in previous studies by the authors (Lima et al 2006, 2009) in which the set-up and measurement procedures are described in detail.

\section{Results and discussion}

\subsection{Characteristics of the microchannel}

Light micrographs of the axisymmetric PDMS microchannels fabricated using the proposed techniques are shown in figures 2 and 3 . The images displayed in figure 2 correspond to the radial cross-sections of three PDMS microchannels obtained using precision wires of diameters $(D) 50 \mu \mathrm{m}$, $75 \mu \mathrm{m}$ and $200 \mu \mathrm{m}$.

We can assess the quality of the radial cross-sectional area of the microfabricated channels using three common shape factors: the roundness (Round), the aspect ratio $(A R)$ and the convexity (Conv). The roundness is defined as Round $=\frac{4 \text { actual area }}{\pi \text { (major axis) }^{2}}$, varies between 0 and 1 and gives an idea of the global deviation of the channel cross-section from a perfect circle. The aspect ratio is defined as $A R=$ minor axis/major axis and is a measure of the cross-section elongation. This factor can have values in the range between 0 and 1, where $A R=1$ corresponds to a shape that is symmetrical in all axes. The convexity is defined here as 


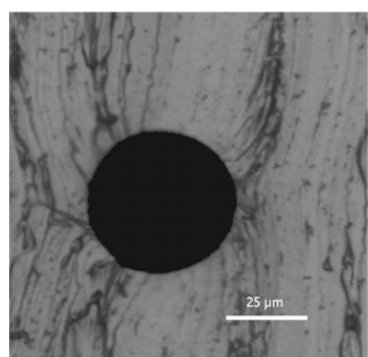

(a)

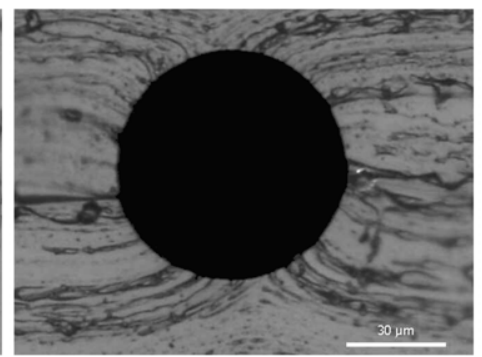

(b)

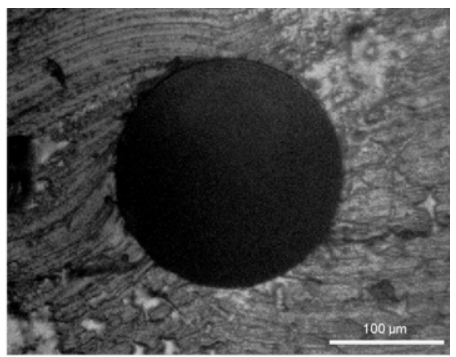

(c)

Figure 2. Radial cross-section of PDMS microchannels with different diameters: (a) $50 \mu \mathrm{m}$; (b) $75 \mu \mathrm{m}$ and (c) $200 \mu \mathrm{m}$.

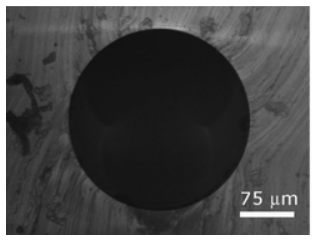

(a)

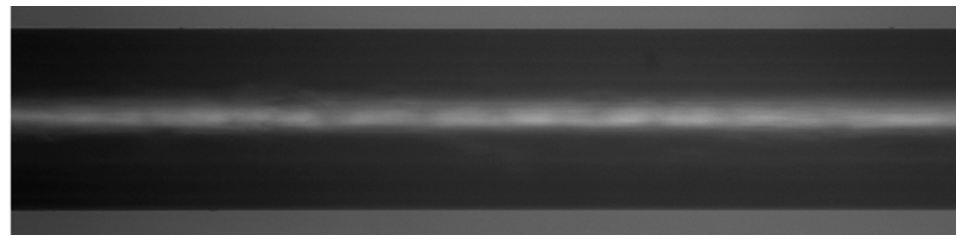

(b)

Figure 3. Radial (a) and axial (b) cross-section of a PDMS microchannel $250 \mu \mathrm{m}$ in diameter.

the ratio between the area of the actual cross-section and the corresponding convex hull area, and highlights the spikiness of the edge defining the cross-section. This factor can also vary between 0 and 1 , where an irregular shape has a convexity close to 0 and a perfectly smooth shape corresponds to Conv $=1$.

The shape factors were evaluated using ImageJ and the ranges of values measured for all channels fabricated using our technique are shown in table 1.

In figure 3 , in addition to the radial cross-section, we show the corresponding axial cross-section for a microchannel with $D=250 \mu \mathrm{m}$. By tuning the fabrication materials and conditions we were able to obtain straight channels without unwanted local bends and with smooth walls. Furthermore, the uniformity of the cross-section is maintained throughout the whole length of the channel, e.g. the image shown in figure $3(b)$ covers $2565 \mu \mathrm{m}$ in length, but the actual size of the channel was greater than $3 \mathrm{~cm}$. In terms of the materials tested we have observed that channels obtained with optical fibers (silicon) present the best overall quality in terms of uniformity of the channel, yielding also the best results for the shape factors (table 1).
As can be observed, the proposed fabrication process can produce good quality, transparent in vitro, real-scale models with diameters comparable to in vivo microcirculatory vessels (6-300 $\mu \mathrm{m})$. Furthermore, this kind of microchannel has other remarkable properties such as good optical transparency, biocompatibility and permeability to gases. This latter property makes these microchannels adequate to culture living cells on their surfaces, such as endothelial cells (Kaji et al 2006, 2007). Further work to pattern endothelial cells on the surfaces of the proposed circular PDMS microchannels is currently under way. By culturing endothelial cells within the microfluidic device, we expect to develop a flow system that resembles in vivo environment and also to identify and quantitatively measure cellular and molecular events occurring between the blood cells and endothelium.

Table 2 summarizes the advantages of axisymmetric channels fabricated using the present technique versus the more commonly used glass capillaries and rectangular PDMS channels. On nearly all accounts, the axisymmetric PDMS channels yield a more realistic representation of real blood vessels, the exception being the current difficulty in generating well-defined complex structures (bifurcations, branches, etc).

Table 2. The most relevant advantages and disadvantages of using glass capillaries, a rectangular PDMS microchannel and a circular PDMS microchannel.

\begin{tabular}{|c|c|c|c|}
\hline $\begin{array}{l}\text { Physicochemical factors that affect } \\
\text { the blood flow behavior }\end{array}$ & $\begin{array}{l}\text { Glass } \\
\text { capillaries }\end{array}$ & $\begin{array}{l}\text { Rectangular PDMS } \\
\text { microchannel }\end{array}$ & $\begin{array}{l}\text { Circular PDMS } \\
\text { microchannel }\end{array}$ \\
\hline Complexity of the geometry & Complex & Complex & Simple \\
\hline Cross-sectional area & Circular & Rectangular & Circular \\
\hline Resistance to the inside pressure & High & Low & High \\
\hline Mechanical properties & Rigid & Flexible & Flexible \\
\hline Ability to culture cells on the inner surface & Not possible & Possible & Possible \\
\hline Ability to study microcirculation pathogenesis phenomena & Not possible & Possible & Possible \\
\hline Cost of the fabrication process & High & High & Low \\
\hline
\end{tabular}




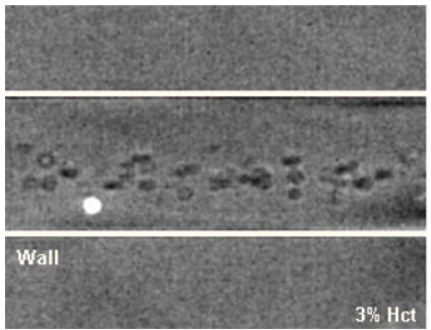

(a)

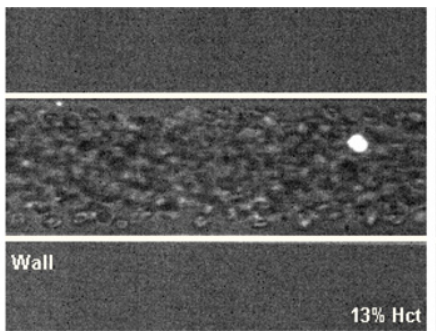

(b)

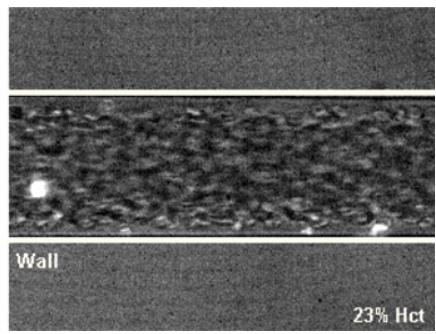

$(c)$

Figure 4. Confocal images obtained at the middle plane of a $75 \mu \mathrm{m}$ channel using a $20 \times$ objective lens (1.6 zoom) for $(a) 3 \%$ Hct, $(b) 13 \%$ Hct and (c) $23 \%$ Hct. The unlabeled RBCs appear as dark gray ellipsoids and the labeled RBCs appear as bright spots.

Thus, future developments in the fabrication of in vitro microvessels should attempt to circumvent this limitation.

\subsection{Cell-free layer visualization}

The circular channels used in this work are cast into a parallelepiped block of PDMS with flat external walls, which allows us to circumvent refraction problems that may occur has a consequence of refraction of light passing through the microchannel wall. The thin layer of PDMS across which the flow is imaged provides an optical window which has a refractive index (of $\sim 1.4-1.5$ depending on the curing conditions (Chang-Yen et al 2005)) similar to that of the solutions used in this work (which may vary between $\sim 1.33$ and 1.4 depending on the concentration of RBCs and its aggregation (Xu et al 2003)). This technique for refractive index matching together with the optical sectioning ability of the confocal system made it possible to obtain a series of optical sectioned images of a reasonable quality at the desired geometrical plane. Figure 4 shows both labeled (laser-emitted light) and unlabeled RBCs (halogen illumination) at different Hcts- $3 \%, 13 \%$ and $23 \%$. In figure 4 it is possible to observe the well-known Faharaeus and Faharaeus-Lindqvist effects (Chien et al 1984) in which a cell-free layer forms close to the wall reducing the local Hct and consequently the local viscosity. Furthermore, preliminary results obtained using a manual tracking MtrackJ plugin from ImageJ indicate that the thickness of the marginal cell-free layer tends to increase with decreasing Hct, which corroborates the results obtained using narrow glass microtubes (Maeda 1996). The cell-free layer is an important phenomenon that happens in microvessels as it reduces the friction between the blood cells and endothelial cells and consequently the flow resistance.

\subsection{RBC displacement and lateral dispersion coefficient}

By analyzing a series of time-consecutive images it is possible to track the path of the labeled RBCs. Figure 5 shows the pathlines of individual labeled RBCs flowing through a $75 \mu \mathrm{m}$ PDMS microtube for a feed Hct of 3\%, $13 \%$ and $23 \%$. The measurements were performed at the center plane for a constant low flow rate $\left(10^{-12} \mathrm{~m}^{-3} \mathrm{~s}^{-1}\right)$. It is clear that RBCs in flowing blood exhibit a random-like transverse motion (i.e. random transverse fluctuations superimposed on the main axial flow) with the magnitude of the fluctuations depending on Hct.
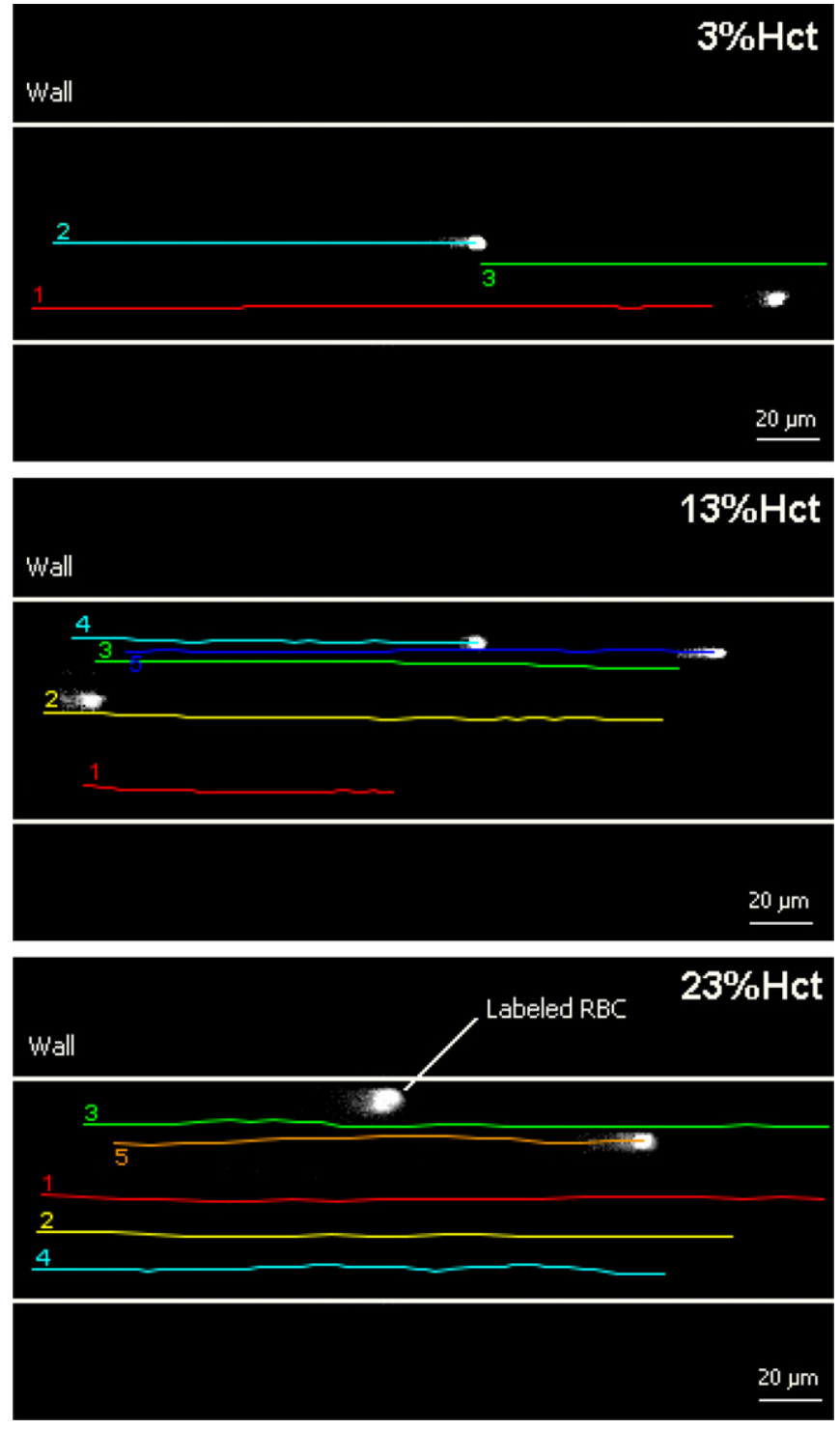

Figure 5. RBCs streamlines for 3\% Hct, 13\% Hct and 23\% Hct.

These qualitative results evidence that for solutions with higher RBC concentrations (e.g. 23\% Hct) the paths of flowing RCBs exhibit higher erratic displacements in the direction normal to the flow. Hence, by measuring the lateral displacements of various labeled RBCs flowing through the microchannel as a function of time, it was possible to calculate 


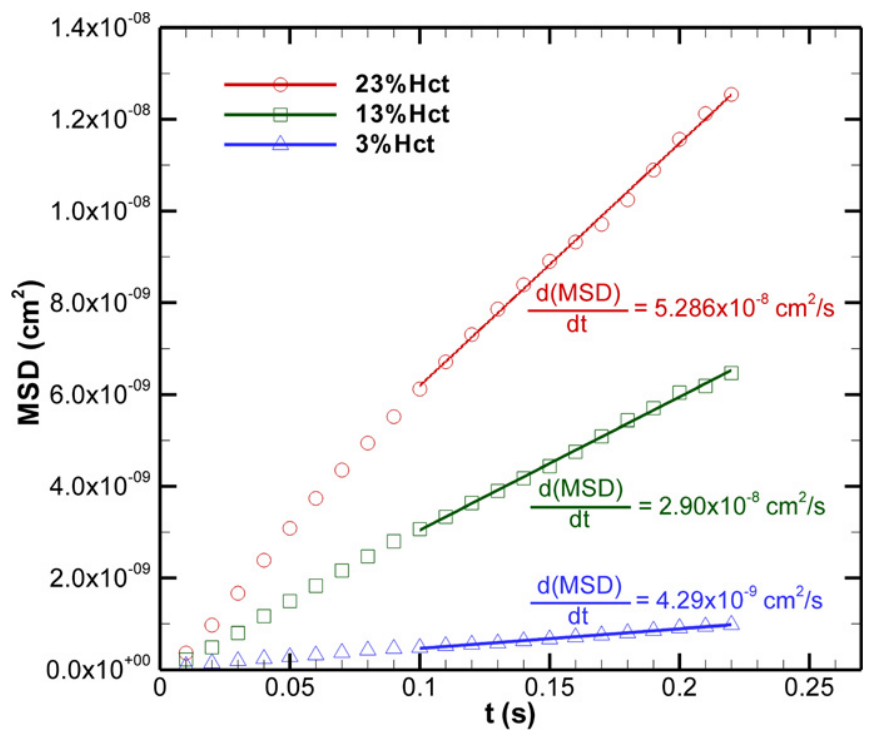

Figure 6. RBC mean square displacement (MDS) at the middle plane of a $75 \mu \mathrm{m}$ circular PDMS microchannel for 3\% Hct, $23 \%$ Hct and $23 \%$ Hct.

the ensemble average of the mean square displacement (MSD) and the corresponding lateral dispersion coefficient, given by

$$
D_{y y}(t)=\frac{1}{N} \sum_{i=1}^{N} \frac{\left\langle\left(R_{i, y}(t)-R_{i, y}(0)\right)^{2}\right\rangle}{2 t},
$$

where $t$ is the time interval, $N$ is the total number of measured RBCs, $\left\langle\left(R_{i, y}(t)-R_{i, y}(0)\right)^{2}\right\rangle$ is the MSD and $R_{i, y}(t)$ is the radial position of cell $i$ at time $t$. Figure 6 illustrates the RBC mean square displacement at the middle plane for several Hcts $(3 \%$, $13 \%$ and $23 \%$ ).

For sufficiently long times, the MSD increases linearly with time and as a consequence the dispersion coefficient attains a constant value, which is characteristic of a diffusive regime. In this regime, a $\mathrm{RBC}$ experiences multiple collisions and the RBC motions can be described as random walks and the spreading is diffusive. The slope of the linear fit to this part of the curve was taken to calculate the corresponding dispersion coefficient. The results of $D_{y y}$ for each Hct are shown in figure 7. For comparison we also show in figure 7 the values of $D_{y y}$ available in the literature corresponding to classic glass capillaries with similar Hct and different diameters (Lima et al 2008b, 2009). As it can be seen, our results are consistent with those in the literature, with $D_{y y}$ strongly depending on Hct and the channel diameter. The RBC $D_{y y}$ increases with Hct, and for $23 \% \mathrm{Hct}$, it is almost one order of magnitude greater than the $D_{y y}$ with $3 \% \mathrm{Hct}$, i.e., the RBCs flowing in a crowded environment tend to undergo multi-body collisions which increases the amplitude of the RBC's lateral motion. As a result, $\mathrm{RBCs}$ at moderate and high concentrations $(13 \% \mathrm{Hct}$ and $23 \% \mathrm{Hct}$ ) tend to exhibit higher erratic radial displacement compared to dilute suspensions of RBCs (3\% Hct).

\section{Conclusions}

In this work, we describe a wire casting technique to fabricate axisymmetric microfluidic channels, with diameters in the

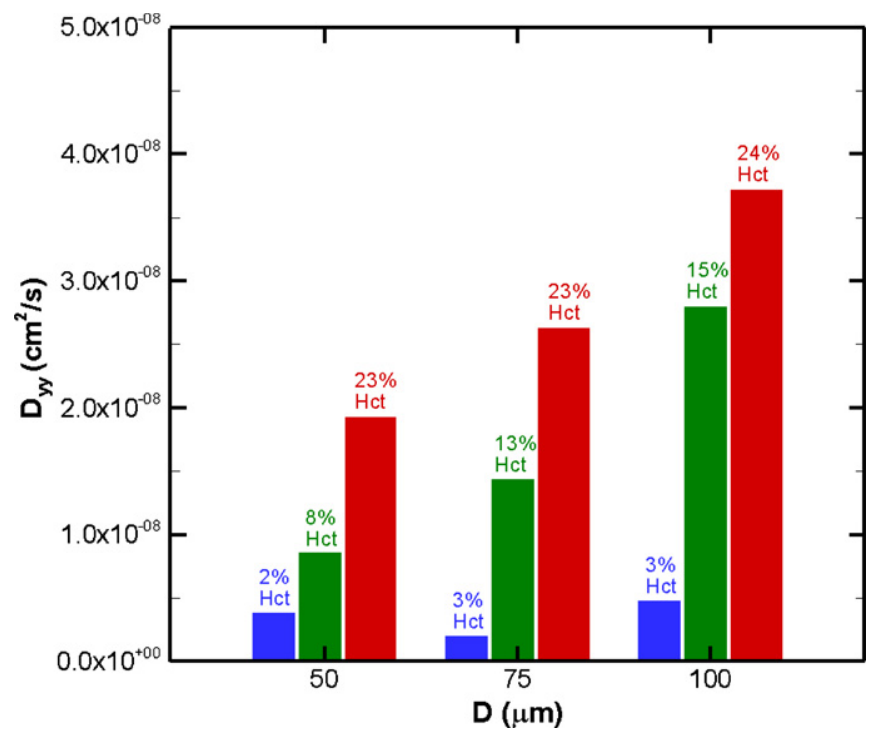

Figure 7. Effect of hematocrit on the RBC dispersion coefficient $\left(D_{y y}\right)$ at the center plane for a $75 \mu \mathrm{m}$ circular PDMS channel. Also shown are the results corresponding to classic glass capillaries with similar Hct and different diameters (Lima et al 2008b, 2009).

range of 50-250 $\mu \mathrm{m}$, suitable for in vitro hemodynamic studies. The method is rapid, inexpensive and requires no clean room or specialized equipment, affording a straightforward way to control the channel dimensions. The channels, fabricated using precision wires of different materials, are made of PDMS, which confers good optical access and biocompatibility, and offers the possibility of culturing endothelial cells in the channel wall. These properties, which will allow one to obtain channels of circular cross-section with non-rigid walls and an irregularly shaped endothelial inner surface, constitute a significant advance in the development of more realistic models of microvessels. Furthermore, the results demonstrate that the confocal system can be successfully integrated with the proposed fabrication technique. This combination provides a powerful way of investigating several microcirculation phenomena and consequently will help to understand the actual physiopathological phenomena in microvessels.

\section{Acknowledgments}

This study was supported in part by the following grants: International Doctoral Program in Engineering from the Ministry of Education, Culture, Sports, Science and Technology of Japan (MEXT) and Grant-in-Aid for Scientific Research (S) from the Japan Society for the Promotion of Science (JSPS; no 19100008). We also acknowledge the support from the 2007 Global COE Program 'Global NanoBiomedical Engineering Educationand Research Network'. We would also like to thank Dr Motohiro Takeda from Tohoku University for providing the blood samples and Dr Paulo André for supplying some of the optical fibers tested in this work. 


\section{References}

Abramoff M, Magelhaes P and Ram S 2004 Image processing with image J. Biophotonics Int. 11 36-42

Q1 Brown D and Larson R 2001 Improvements to parallel plate flow chambers to reduce reagent and cellular requirements $B M C$ Immunol. 2-9

Chang-Yen D A, Eich R K and Gale B K 2005 A monolithic PDMS waveguide system fabricated using soft-lithography techniques J. Lightwave Technol. 23 2088-93

Q2 Chien S, Usami S and Skalak R 1984 Blood flow in small tubes Handbook of Physiology-The Cardiovascular System vol. IV pp 217-49

Fujiwara H, Ishikawa T, Lima R, Matsuki N, Imai Y, Kaji H, Nishizawa M and Yamaguchi T 2009 Red blood cell motions in high-hematocrit blood flowing through a stenosed microchannel J. Biomech. 42 838-43

Goldsmith $\mathrm{H}$ and Turitto V 1986 Rheological aspects of thrombosis and haemostasis: basic principles and applications. ICTH Report-Subcommittee on Rheology of the International Committee on Thrombosis and Haemostasis Thromb. Haemost. 55 415-35

Kaji H, Hashimoto M and Nishizawa M 2006 On-demand patterning of protein matrixes inside a microfluidic device Anal. Chem. 78 5469-73

Kaji H, Sekine S, Hashimoto M, Kawashima T and Nishizawa M 2007 Stepwise formation of patterned cell co-cultures in silicone tubing Biotechnol. Bioeng. 98 919-25

Q3 Lima R et al 2009 Measurement of individual red blood cell motions under high hematocrit conditions using a confocal micro-PTV system Ann. Biomed. Eng. submitted

Lima R, Ishikawa T, Imai Y, Takeda M, Wada S and Yamaguchi T 2008b Radial dispersion of red blood cells in blood flowing through glass capillaries: role of haematocrit and geometry J. Biomech. 41 2188-96

Lima R, Wada S, Takeda M, Tsubota K and Yamaguchi T 2007 In vitro confocal micro-PIV measurements of blood flow in a square microchannel: the effect of the haematocrit on instantaneous velocity profiles $J$. Biomech. 40 2752-7

Lima R, Wada S, Tanaka S, Takeda M, Ishikawa T, Tsubota K, Imai Y and Yamaguchi T 2008a In vitro blood flow in a rectangular PDMS microchannel: experimental observations using a confocal micro-PIV system Biomed. Microdevices 10 153-67

Lima R, Wada S, Tsubota K and Yamaguchi T 2006 Confocal micro-PIV measurements of three dimensional profiles of cell suspension flow in a square microchannel Meas. Sci. Technol. 17 797-808

Maeda N 1996 Erythrocyte rheology in microcirculation Jpn. J. Physiol. 46 1-14

McDonald J C, Duffy D C, Anderson J R, Chiu D T, Wu H K, Schueller O J A and Whitesides G M 2000 Fabrication of microfluidic systems in poly(dimethylsiloxane) Electrophoresis $2127-40$

Meijering E, Smal I and Danuser G 2006 Tracking in molecular bioimaging IEEE Signal Process. Mag. 23 46-53

Oliveira M S N, Alves M A, Pinho F T and McKinley G H 2006 Viscous fluid flow through microfabricated hyperbolic contractions Exp. Fluids 43 437-51

Oliveira M S N, Rodd L E, McKinley G H and Alves M A 2008 Simulations of extensional flow in microrheometric devices Microfluidics Nanofluidics 5 809-26

Pries A, Secomb T, Gessner T, Sperandio M, Gross J and Gaehtgens P 1994 Resistance to blood flow in microvessels in vivo Circ. Res. 75 904-15

Shevkoplyas S, Gifford S, Yoshida T and Bitensky M 2003 Prototype of an in vitro model of the microcirculation Microvasc. Res. 65 132-6

Suzuki Y, Tateishi N, Soutani M and Maeda N 1996 Deformation of erythrocytes in microvessels and glass capillaries: effects of erythrocyte deformability Microcirculation 3 49-57

Takeuchi S, Garstecki P, Weibel D B and Whitesides G M 2005 An axisymmetric flow-focusing microfluidic device Adv. Mater. 17 1067-72

Wang G-J, Ho K-H, Hsu S-H and Wang K-P 2007 Microvessel scaffold with circular microchannels by photoresist melting Biomed. Microdevices 9 657-63

Xu X, Wang R K, Elder J B and Tuchin V V 2003 Effect of dextran-induced changes in refractive index and aggregation on optical properties of whole blood Phys. Med. Biol. 48 1205-21 


\section{Queries}

(1) Author: Please provide volume number in reference 'Brown and Larson 2001'.

(2) Author: Please provide the name and place of publication in reference 'Chien et al 1984'.

(3) Author: Please update reference 'Lima et al 2009'.

(4) Author: Please be aware that the colour figures in thisa rticle will only appear in colour in the Web version. If you require colour in the printed journal and have not previously arranged it, please contact the Production Editor now.

\section{Reference linking to the original articles}

References with a volume and page number in blue have a clickable link to the original article created from data deposited by its publisher at CrossRef. Any anomalously unlinked references should be checked for accuracy. Pale purple is used for links to e-prints at arXiv. 